Información Tecnológica

Vol. 29(3), 229-236 (2018)

http://dx.doi.org/10.4067/S0718-07642018000300229

\title{
Campos Electromagnéticos No lonizantes para la Inocuidad Alimentaria
}

\author{
Arturo B. Rodriguez ${ }^{(1)}$, Angélica Ganga ${ }^{(2)}$ y Liliana Godoy (2) \\ (1) Universidad Santiago de Chile, Departamento de Tecnología Industrial, Grupo de Investigación en Nuevas \\ Tecnologías (GINT), Santiago-Chile. (e-mail: arturo.rodriguez@usach.cl) \\ (2) Universidad Santiago de Chile, Departamento de Ciencia y Tecnología de los Alimentos, Laboratorio de \\ Biotecnología y Microbiología Aplicada (LAMAP). Santiago-Chile. (e-mail: angelica.ganga@usach.cl; \\ liliana.godoy@usach.cl)
}

Recibido Sep. 8, 2017; Aceptado Nov. 20, 2017; Versión final Feb. 5, 2018, Publicado Jun. 2018

\begin{abstract}
Resumen
Se realizó un estudio sobre los efectos e influencia que tienen los campos electromagnéticos convencionales (Wifi y Celular) sobre microorganismos contaminantes. El estudio se realizó utilizando medios Luria-Bertani (LB) y Agua Peptonada 2,5\% (WP), en dos canales de la banda Wifi (1 y 11), utilizando muestras de control en cada caso, por un periodo de 2 días a $-30 \mathrm{dBm}$. Los resultados muestran un significativo descenso en la viabilidad de Escherichia coli ATCC25922 crecida en WP. Sin embargo, no se encontró cambios significativos cuando se utilizó LB; ambos resultados se repiten para ambos canales. Esto generará posibles tecnologías anticontaminantes para la industria alimentaria, utilizando como principio de funcionamiento los campos electromagnéticos.
\end{abstract}

Palabras Clave: Escherichia; microorganismos; campos electromagnéticos; Wifi; banda no-inonizante

\section{Non-Ionizing Electromagnetic Fields for Food Safety}

\section{Abstract}

A study was done on the effects and influence of conventional electromagnetic fields (Wifi and Cellular) on contaminating microorganisms. The study was performed using Luria-Bertani (LB) and Water Peptone $2.5 \%$ (WP) media in two Wifi band channels (1 and 11) using control samples in each case, for a period of 2 days at $-30 \mathrm{dBm}$. The results show a significant decrease in the viability of Escherichia coli ATCC25922 over WP. However, no significant changes were found when LB was used; both results are repeated for both channels. This generates possible anti-pollution technologies for the food industry using electromagnetic fields as the operating principle.

Keywords: Escherichia; microorganism; electromagnetic fields; Wifi; non-ionizing band 


\section{INTRODUCCIÓN}

El estudio de los microorganismos y su efecto contaminante es foco de investigación en la comunidad científica, sobre todo por su importancia en el área de la inocuidad alimentaria. La tecnología WireLess comúnmente denominada WIFI, está sustentada en la velocidad de transmisión proporcionada por la banda 2,4 - 2,482 Ghz, por lo general desde los $-30 \mathrm{dBm}$ hasta los $-70 \mathrm{dBm}$, después de esa potencia la información es presa del ruido y la relación señal a ruido disminuye drásticamente perjudicando la recepción. Los seres humanos estamos expuestos a rangos variables de potencia en la banda antes mencionada, entre otras. Además, estamos expuestos a diferentes canales que no son sujeto de estudio en el presente artículo.

La exposición de los seres humanos a radiofrecuencia (RF) ha ido incrementándose con el avance de las telecomunicaciones, por lo cual el mundo vive un continuo sometimiento radiativo. En general, estamos expuestos a radiatividad No lonizante, es decir aquellas que no modifican la estabilidad atómica del objeto expuesto. La exposición actual es entre 0,1 y 5 Ghz que pertenece a la mencionada banda No lonizante.

Por otra parte, la reglamentación sanitaria cada vez es más exigente por lo que la inocuidad alimentaria es de gran interés y la industria exige metodologías cada vez más eficaces y rápidas, debido a que sus efectos en la productividad y el riesgo sanitario deben ser reducidos al mínimo. La comunidad científica y la industria alimentaria se encuentran en la búsqueda de soluciones que permitan la sanitización eficiente y efectiva de los procesos industriales. Para ello, se han utilizado diferentes métodos donde se incorporan componentes químicos y físicos, como por ejemplo el uso de ozono, UV, entre otros (Sartori et al., 2007; Moreno et al., 2011). Estas metodologías son utilizadas con cierto cuidado por las posibles consecuencias negativas que traen. Sin embargo, la utilización de una metodología sin material de aporte y sin productos dañinos como consecuencia de la sanitización, son de relevante importancia. En este artículo se estudia el efecto de las ondas electromagnéticas sobre Escherichia coli, uno de los microorganismos contaminantes más importantes para la industria alimentaria, proyectando el uso de éstas como control para la inocuidad.

En la literatura se han reportado resultados de diferentes tipos de materia orgánica expuesta a CEM. (Bates, 1991), observó que la exposición a CEM de baja potencia en la banda de los $50 \mathrm{~Hz}$, se correlaciona con cánceres en el sistema nervioso central, especialmente cerebrales. A nivel celular se ha reportado apoptosis que se define como la autoprogramación de muerte celular, así como su efecto sobre la respuesta a daños en el DNA, proliferación celular y alteración general del ciclo celular (Yu-xia et al., 2012), lo cual indicaría que las CEM podrían tener un efecto en la salud humana. Sin embargo, (Karbowski et al., 2012) reportaron lo contrario, observando una inhibición del crecimiento en células cancerígenas cuando son expuestas a CEM.

Similares resultados se han obtenido al exponer células mononucleares de sangre de donadores sanos que fueron expuestas in vitro a una radiatividad de $900 \mathrm{Mhz}$ por 1h/día durante 3 días, mostrando una disminución en la proliferación celular (Capri et al., 1999). Sin embargo, (Lantow et al., 2006) observaron que al exponer células de la línea Mono Mac 6 a 1800 Mhz por 12 h, no mostraron mayor diferencia en su viabilidad cuando fueron comparadas con el ensayo control. Se ha relacionado el efecto de las CEM a nivel celular con la producción de especies reactivas al oxígeno (ROS), las que inducirían a daños oxidativos a nivel del DNA, modificando las bases nitrogenadas o azúcares, así como induciendo al rompimiento de las hebras de DNA, o bien afectando directamente a membranas (Akdag et al., 2013; Dasdag et al., 2016).

Por su parte, a nivel molecular se ha observado una baja regulación de los genes SOD1, SOD2, CAT y GPX1, relacionados con la respuesta a stress oxidativo ( $\mathrm{Ni}$ et al., 2013). En otras líneas investigativas que aportan en la misma dirección se distingue el trabajo de (Sun et al., 2017) que al exponer células de la línea HL 60 (derivadas de la leucemia promielocítica humana) a $900 \mathrm{~Hz}$ por $4 \mathrm{~h} /$ día durante 5 días consecutivos observaron un daño a nivel del DNA mitocondrial.

En el mundo vegetal, se ha investigado el efecto sobre la velocidad de crecimiento, germinación de las semillas, metabolismo de carbohidratos, daños oxidativos, expresión de genes, actividades enzimáticas, entre otros (Halgamuge, 2016). En este sentido, al estudiar el efecto de exposición a 400 y 900 Mhz durante 2 horas en plantas de Lemma minor, se observó una disminución en el crecimiento de la planta, comparada con el control, lo cual podría ser debido a un aumento del stress de ésta, evidenciándose por un aumento en la peroxidación de lípidos y el contenido de $\mathrm{H}_{2} \mathrm{O}_{2}$, acompañado por un disminución de la actividades enzimáticas antioxidantes (Tkalec et al., 2007).

También se demostró que las plantas de maíz al ser expuestas a 940 Mhz tuvieron un incremento en la concentración de malondialdehido así como de la actividad catalasa, siendo ambos indicadores de peroxidación (Zare et al., 2015). Paralelamente, se ha demostrado un retraso en la germinación de semillas de maíz, explicado por la alteración en el contenido de almidón de las mismas, así como un efecto sobre el metabolismo de la sacarosa (Kumar et al., 2016). 
Por su parte, al exponer Arabidopsis thaliana a 1882 Mhz se observó una reducción en el número de cloroplastos como de pigmentos fotosintéticos, lo cual implicó en una reducción del crecimiento de la planta (Stefi et al., 2016). Recientemente, estos mismos autores observaron que al exponer plantas de Gossypium hirsutum (algodón) durante 21 días a 1.882 Mhz se produjo una baja en la producción de biomasa de la planta y raíces, lo que se explicó por la reducción en los pigmento fotosintéticos y la disminución en los tamaños de los cloroplastos (Stefi et al., 2017).

También, al exponer plantas de tomates a 900 Mhz por 10 minutos se observó una sobreexpresión de genes relacionados a stress (calmodulina, inhibidor de proteasa y proteínas de unión al mRNA) evidenciaron un aumento de 4 a 6 veces en la expresión de éstos en comparación a la muestra control (Roux et al., 2006). En resumen, los CEM si afectaría el crecimiento en el mundo vegetal, no estando aún claras sus causas.

En lo que respecta al efecto del CEM sobre los microorganismos se han obtenido resultados contradictorios. De manera de definir el efecto de las torres de teléfonos móviles sobre los microorganismos, se expusieron cultivos de Eschericia coli a diferentes distancias de estas torres, variando así la emisión de radiación (95$57 \mathrm{dBm}$ ). Los resultados mostraron una dramática disminución de la viabilidad del cultivo (Mulye et al., 2015). Por su parte, al someter Klebsiella pneumonia a CEM de 2,4 Ghz por 3; 4,5 y 8 horas se observó una variación en la resistencia del microorganismo a una serie de antibióticos (aztreonam, ceftriaxona, imipenem, piperacilina y cefataxima). Cuando fue expuesto a 3 horas hubo un pequeño aumento en la sensibilidad a estos antibióticos, siendo claramente observado a las 4,5 horas. Sin embargo, a las 8 horas de exposición, $K$. pneumonia aumento su resistencia (Taheri et al., 2017).

Por otro lado, al realizar un estudio similar utilizando ahora cultivos de Listeria monocytogenes y E. coli que fueron expuestos a un CEM en la banda 2,4-2,482 GHz y a diferentes tiempos de exposición, se observó una respuesta diferente de cada especie a los antibióticos. En el caso de E. colise observó una sensibilidad mayor al antibiótico al ser expuesta a 6 horas al CEM, ya que, sobre este tiempo, la bacteria retornó o incluso superó la resistencia observada a tiempo cero.

En el caso de L. monocytogenes no se observó mayor variación con respecto a la sensibilidad de los antibióticos. Ha sido propuesto que los microorganismos pueden inducir respuestas de supervivencia en tiempos definidos de acuerdo con el CEM, conocido como "narrow window" (Taheri et al., 2015). Algunos autores cuestionan los estudios realizados hasta ahora en relación al efecto de los CEM sobre células humanas, plantas y microorganismos, ya que los resultados no son del todo claro, existiendo aún ambigüedad, por lo que es necesario normalizar los protocolos de experimentación, así como la reproducibilidad de los resultados (Belyaev et al., 2016).

\section{METODOLOGIA}

Se procede a describir el microrganismo utilizado en la experimentación y los procesos de preparación para el estudio, para luego describir el procedimiento experimental, detallando el equipo y los experimentos.

\section{Microorganismo}

Se utilizó la cepa E. coli ATCC25922 que fue inicialmente crecida en $5 \mathrm{~mL}$ de medio líquido LB (Luria-Bertani $(0,5 \%$ extracto de levadura, $1 \%$ triptona y $1 \% \mathrm{NaCl}))$ durante $16 \mathrm{~h}$ a $37^{\circ} \mathrm{C}$ con agitación. Luego fue mantenida en agar LB $(0,5 \%$ extracto de levadura, $1 \%$ triptona, $1 \% \mathrm{NaCl}$ y $2 \%$ agar) para su posterior uso.

\section{Experimentación}

Para realizar el estudio se utilizó una cámara de resonancia magnética con tres antenas $\lambda / 2$ dispuestas ortogonalmente, logrando un campo estable a - $40 \mathrm{dBm}$. Dicha cámara fue utilizada para someter a exposición el microorganismo de estudio.

Para ello, fue crecido en $5 \mathrm{~mL}$ de medio LB durante $16 \mathrm{~h}$ a $37^{\circ} \mathrm{C}$ con agitación constante hasta una concentración de $1 \times 10^{8}$ células $/ \mathrm{mL}$. Posteriormente, $1 \times 10^{6}$ células $/ \mathrm{mL}$ fueron inoculadas por triplicado en 5 $\mathrm{mL}$ de medio LB fresco y en $5 \mathrm{~mL}$ de WP (2,5\%) para ser sometidos durante 2 días a una frecuencia de 2,412 Ghz (Canal 1) y 2,462 Ghz (Canal 11) independientemente, considerando como control los cultivos del microorganismo no expuestos, garantizando aislamiento al CEM y bajo la misma temperatura $\left(28^{\circ} \mathrm{C}\right)$. 
Transcurrido 2 días, se realizaron diluciones seriadas en agua peptonada $0,1 \%$. Estas fueron sembradas en alícuotas de $100 \mu \mathrm{L}$ sobre placas agar LB y se incubaron durante $16 \mathrm{~h}$ a $37^{\circ} \mathrm{C}$. Todas las pruebas se hicieron por triplicado y los resultados se obtuvieron por recuento en placa como UFC/mL.

\section{Análisis Estadístico}

El análisis y tratamiento de los resultados fueron realizados en el programa Statgraphics Centurion XVI.I (StatPoint Technologies, Warrenton, USA), mediante el uso de la prueba de múltiple rango para los datos con distribución normal. Se consideró la existencia de diferencias significativas cuando $p \leq 0,05$.

\section{RESULTADOS Y DISCUSIÓN}

Las figuras muestran el recuento en placa de E. coli expuesto a la banda 2,4-2,482 Ghz (WIFI), donde se observan los resultados para los canales 1 y 11 (ver Figura 1 y 2 ). El tiempo de exposición fue de 48 horas y se utilizaron dos medios de cultivo (WP y LB).

Las muestras fueron mantenidas a $28^{\circ} \mathrm{C}$. En la figura 1 se observa, que el eje de recuento microbiológico (vertical) fue divido en dos segmentos con el objetivo de mostrar la diferencia observada en ambos medios de cultivo bajo radiatividad emitida a 2,412 Ghz (Canal 1).

Cuando $E$. coli fue crecida en medio WP el recuento a tiempo final en WIFI (48 Horas) fue menor comparado con el control, mientras que cuando fue crecida en medio LB no se observan diferencias estadísticamente significativas entre control y WIFI en igualdad de condiciones que la anterior.

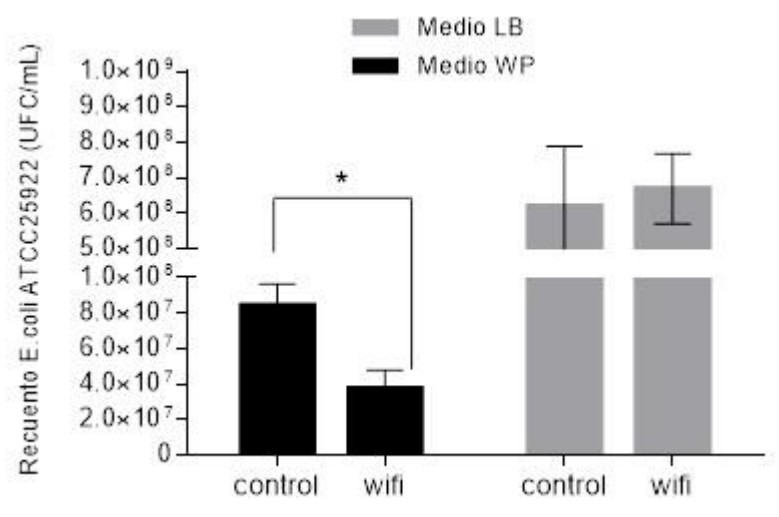

Fig. 1: Crecimiento de E. coli expuesta a 2,412 Ghz (Canal 1). Fuente: Propia

La figura 2 muestra el crecimiento de E. coli en la banda radiante de 2,462 Ghz (Canal 11). Similarmente a lo realizado con la figura 1 , los ejes fueron divididos en dos segmentos. Además, se observa de igual forma que en el ensayo anterior, que cuando $E$. coli fue crecida en medio WP el recuento a tiempo final en WIFI (48 Horas) fue menor comparado con la muestra control, los resultados en LB son similares al canal anterior. Como es posible apreciar, con estos resultados se evidencia que el efecto del CEM sobre el crecimiento bacteriano depende del medio de cultivo utilizado.

En el caso de este experimento se utilizó LB que es un medio que posee extracto de levadura, triptona y $\mathrm{NaCl}$, una composición más rica en nutrientes que el medio WP que sólo posee peptona, lo que determina la existencia de mayor cantidad de moléculas de agua libre por unidad de volumen.

Los resultados del presente estudio son similares a los reportados por (Akbal et al., 2013), quienes estudiaron los efectos antibacterianos de las ondas electromagnéticas sobre E. coli y Bacillus subtilis.

Ambos cultivos fueron expuestos a 1,800 Mhz entre $1 \mathrm{a} 10 \mathrm{~h}$, observando que el crecimiento de $E$. coli disminuye, en comparación con el ensayo control (sin exposición), nótese la cercanía de las bandas de frecuencia utilizadas con el presente trabajo. Asimismo, el crecimiento de $B$. subtilus también se vio levemente afectado. 


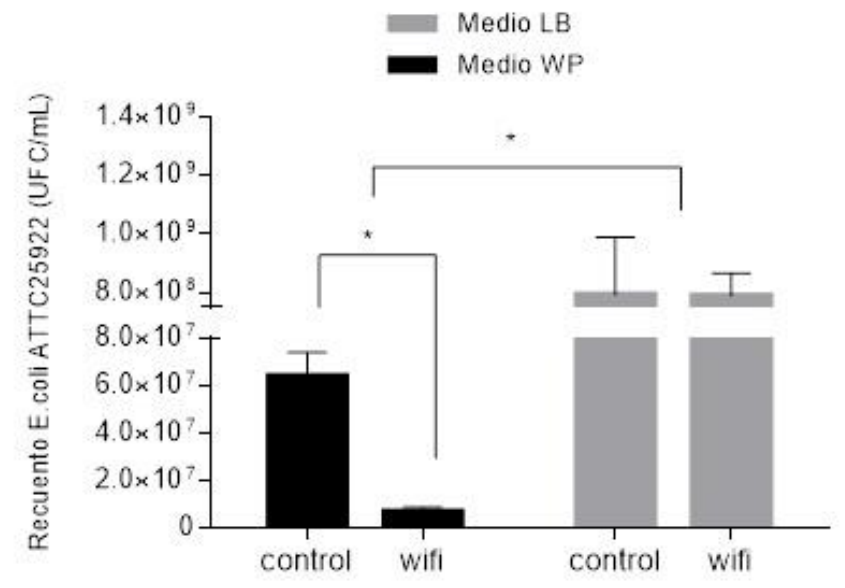

Fig. 2: Crecimiento de E. coli expuesta a 2,462 Ghz (Canal 11). Fuente: Propia

En la figura 3, se observa la comparación de los recuentos de las muestras expuestas a radio frecuencia Wifi de ambos canales y ambos medios. Donde se observa la tendencia a un menor recuento en aquellas expuestas a mayor frecuencia en medio WP.

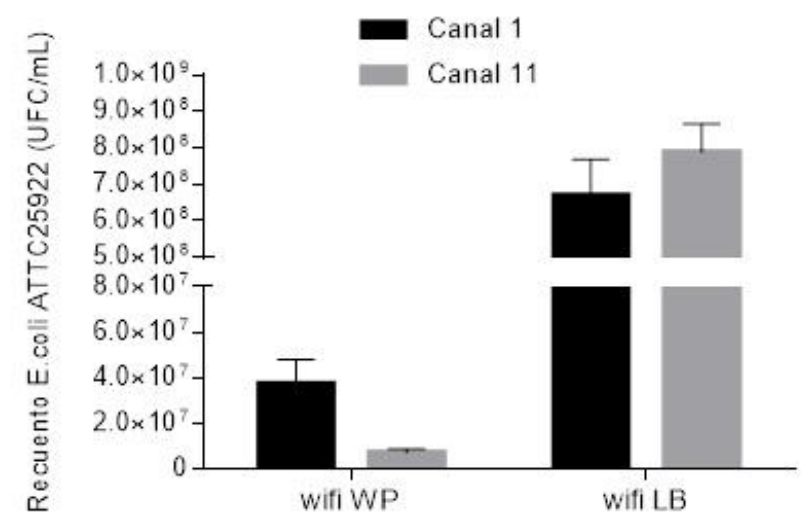

Fig. 3: Comparación del efecto de la radio frecuencia WIFI sobre el crecimiento de E. coli en ambos canales y medios de cultivo. Fuente: Propia

Si bien existen pocos estudios que evalúen cómo los CEM afectan los sistemas biológicos, se ha reportado que la intensidad y las características espaciales de éste, son los que se relacionan con el efecto sobre el crecimiento de los microorganismos.

EI CEM produce rotación de los dipolos magnéticos restringiendo su movilidad, lo que tiene un efecto sobre la velocidad de las reacciones bioquímicas producidas al interior de los microorganismos (Motta et al., 2001).

Asimismo, debido a su composición electrolítica los organismos vivos son por lo general conductores de la electricidad. A través de las membranas celulares y de los fluidos intra y extracelulares existen corrientes iónicas, a las cuales debe estar asociado un campo magnético.

Además, en los sistemas biológicos existen estructuras magnéticamente influenciables como los radicales libres que presentan propiedades paramagnéticas y aquellas en las que intervienen sustancias ferromagnéticas. La respuesta de un sistema biológico a un campo magnético externo depende tanto de las propiedades magnéticas intrínsecas del sistema como de las características del campo externo y de las propiedades del medio en el cual tiene lugar el fenómeno.

Esto permite que los organismos vivos al someterse a campos electromagnéticos respondan de diferentes formas. Debido a esta respuesta el equipo de investigación espera desarrollar una técnica que permita la eliminación total o diminución significativa de bacterias que son comunes e importantes en la sanitización e inocuidad en los procesos de la industria alimentaria. 


\section{CONCLUSIONES}

El ensayo experimental donde se utilizó radiatividad no ionizante en la banda 2,4-2,482 GHz, para tal efecto la cámara electromagnética confino el campo en una zona dando estabilidad al campo durante la experiencia y fue realizado bajo condiciones estrictamente controladas y muestran la existencia de un efecto sobre el crecimiento de E. coli ATCC25922. Las diferencias encontradas en ambas bandas radiativas, muestran un efecto inhibitorio sobre el crecimiento de este microorganismo en medio WP a diferencia de lo observado en medio LB donde no se evidencia el efecto inhibitorio. Desde la perspectiva del medio de cultivo, se diferencian en la presencia de un componente que en este caso es extracto de levadura el cual es soluble formado por la autolisis de levaduras y que contiene alto niveles de vitaminas aminoácidos y factores de crecimiento. Por otro lado, desde la cantidad de agua libre presente en los medios, el medio WP contendría por, su composición química, más que LB. Esto sugiere que las razones asociadas al fenómeno observado podrían ser a la mayor cantidad de agua disponible en uno de los medios

\section{AGRADECIMIENTOS}

Especial agradecimiento al Proyecto Fortalecimiento Usach USA1799_GM181622 y el Programa Dicyt de la Universidad de Santiago de Chile, por su importante apoyo al desarrollo de la investigación.

\section{REFERENCIAS}

Akbal A. y Balik H., "Investigation of Antibacterial Effects of Electromagnetic Waves Emitted by Mobile Phones", Pol. J. Environ. Stud., 22(1), 1589-1594 (2013).

Bates M., "Extremely low frecuency electromagnetic fields and cancer: the epidemiologic evidence", Environmental Health Perspectives, 95(1), 147-156 (1991).

Belyaev I., Dean A., Eger H., Hubmann G., Jandrisovits R., Kern M., Kundi M., Moshamme H., Lercher P., Müller K., Oberfeld G., Ohnsorge P., Pelzmann P., Scheingrabe C. y Thill R., "EUROPAEM EMF Guideline 2016 for the prevention, diagnosis and treatment of EMF-related health problems and illnesses", Review Environmental and Health, 31(1), 363397 (2016).

Capri M., Salvioli S., Altilia S., Sevini F., Remondini D., Mesirca P., Bersani F., Monti F., y Franceschi C. "Age-dependent effects of in vitro radiofrequency exposure (mobile phone) on CD95+ Thelper human lymphocytes", Ann. NY Acad. Sci., 1067(1), 493-499 (1999).

Halgamuge M., "Review: wak radiofrequency radiation exposure from mobile phone radiation on plants. Electromagnetic biology and medicine", http:// dx.doi.org/10.1080/15368378.2016.1220389, 36(2), 213-235(2016).

Karbowski, L., Harribance, S., Buckner, C., Mulligan, B., Koren, S., Lafrenie, R. y Persiger, M., "Digitized quantitative electroencephalographic patterns appliend as magnetic fields inhibit melanoma cell a proliferation in culture", http://.doi.org/10.1016/J.neulet.2012.06.059, Neuroscience Letter, 523(2), 131-134(2012).

Kumar A., Pal-Singh H., Batish D., Kaur S. y Kumar-Kohl R., "EMF radiations (1800 MHz)-inhibited early seedling growth of maize (Zea mays) involves alterations in starch and sucrose metabolism", Protoplasma. 253(4), 1043-1049(2016).

Lantow M., Viergutz T., Weiss D. G. y Simko M., "Comparative study of cell cycle kinetics and induction of apoptosis after exposure of human mono Mac 6 cells to radiofrequency radiation", Radiat. Res., 166(3), 539-543 (2006).

Moreno, A., Castro, C., Centeno, A. y Giraldo, S., "Cinética de la Desinfección Fotocatalítica de Agua contaminada con E. coli: Efecto de la Concentración del Fotocatalizador y la Potencia de Irradiación”, Inf. tecnol., 22(3), 69-78 (2011).

Motta, M. A., Montenegro, E. J. N.; Stamford, T. L. M.; Silva, A. R. y Silva, F. R. "Changes in Saccharomyces cerevisiae development induced by magnet fields", Biotechnology Progress, 17, 970 - 973 (2001).

Mulye K., Thorat V., Talker J., Talker E. y Pawar, J., "Effect of electromagnetic radiations emitted by mobile towers on survival of E. coli. IOSR Journal of Environmental Sciencia", Toxicology and Food Technology, 9(12), 31-34 (2015).

Ni S., Yu Y., Zhang Y., Wu W., Lai K., Yao K. "Study of Oxidative Stress in Human Lens Epithelial Cells Exposed to 1.8 GHz Radiofrequency Fields”, PLoS ONE., 8(8), e72370 (2013).

Roux D., Vian A., Gitad S., Bonnet P., Paladian F., Davies E. y Ledoigt G., "Electromagnetic fields (900 MHZ) evoke consitent molecular response in tomato plants", Physiologia Plantarum, 128(1), 283-288 (2006).

Sartori, L.; Luiz A. y Schulz, H., "Adecuación de la Calidad Microbiológica de Desechos de Lagunas de Estabilización por la Aplicación de Ozono", Inf. tecnol., 18(6), 83-92 (2007).

Stefi A., Margaritis L. y Christodoulakis N., "The effect of the non ionizing radiation on cultivated plants of Arabidopsis thaliana (Col.)", Flora, 223(1), 114-120 (2016).

Stefi, A.,Margaritis, L. y Christodoulakis N., "The effecto of the non iionizing radiation on expose, laboratory cultuved unpland cotton (Gossypium hirsutum L) plants". Flora, 226, 55-64(2017).

Sun Y., Zonga L., Gaoa Z., Zhub S., Tonga J. y Caoa Y., "Mitochondrial DNA damage and oxidative damage in HL-60 cells exposed to $900 \mathrm{MHz}$ radiofrequency fields", doi: 10.1016/j.mrfmmm.2017.03.001, Mutation Research, 797-799, 7-14 (2017). 
Taheri M., Monrtazavi S., Moradi M., Mansouri Sh., Nouri F., Mortazavi S. y Bahmanzadegan F., "Klebsiella pneumonia, a Microorganism that Approves the Non-linear Responses to Antibiotics and Window Theory after Exposure to Wi-Fi 2.4 GHz Electromagnetic Radiofrequency Radiation", J Biomed Phys Eng., 5(3),115-20 (2015).

Taheri M., Moradi M., Mortazavi S., Mandouri S., Hatam G. y Nouri F., "Evaluation of the $900 \mathrm{MHz}$ radiofrequency radiation effect on the antimicrobial susceptibility and growth rate of Kleibiella pneumoniae", Shizar E-Medi J., 18(3), e 444946 (2017).

Tkalec M., Malarić K. y Pevalek-Kozlina B., "Exposure to radiofrequency radiation induces oxidative stress in duckweed Lemna minor L", Science of The Total Environment. 338(1-3), 78-89 (2007).

Yu-xiao L., Jun-li T., Guo-qing L., Zhi-wen Z., Jing-hui X., Hong-sheng L., Heng Z., Ji-de C., Yuan-ling L., An-ming L. y Yi Z., "Exposure to 1950-MHz TD-SCDMA electromagnetic fields affects the apoptosis of astrocytes via caspase-3-dependent pathway", PLoS One., 7(8), e42332 (2012).

Zare H., Mohsenzadeh S., Moradshahi A., "Electromagnetic Waves from GSM Mobile Phone Simulator and Abiotic Stress in Zea mays L.”, doi: 10.4172/2155-9600.S11-003, J. Nutr. Food. Sci., S11:003 (2015). 
